\title{
Feasibility study of dark matter searches with the CUORE experiment
}

\section{Vignati*, on behalf of the CUORE collaboration}

Sapienza Università di Roma and INFN Sezione di Roma, Roma, I-00185, Italy

E-mail: marco.vignati@romal.infn.it

CUORE will be a 1 ton experiment made of about $1000 \mathrm{TeO}_{2}$ bolometers. It will probe the neutrinoless double beta decay $(0 v \mathrm{DBD})$ of ${ }^{130} \mathrm{Te}$, a tool to test the neutrino nature and mass. The excellent energy resolution and the low background of these detectors will make CUORE a leading experiment in this field, improving the sensitivity to the half-life of $0 v \mathrm{DBD}$ by more than an order of magnitude. Bolometric detectors, however, are also sensitive to nuclear recoils and can be used to search for dark matter interactions. In principle CUORE, thanks to its mass, could look for an annual modulation of the counting rate at low energies. We developed a trigger and a pulse shape identification algorithm, that allow to lower the energy threshold down to the few $\mathrm{keV}$ region. We present the preliminary results obtained on an array made of four CUORE-like crystals, and the prospects for a dark matter search in CUORE.

Identification of Dark Matter 2010-IDM2010

July 26-30, 2010

Montpellier France

\footnotetext{
*Speaker.
} 


\section{The CUORE experiment}

The CUORE experiment is designed to search for the neutrinoless double beta decay ( $0 v \mathrm{DBD})$ of ${ }^{130} \mathrm{Te}$ [1]. The observation of this nuclear decay would determine that the neutrino is a Majorana particle, unlike all other fermions that are Dirac particles, and would set a scale for the absolute value of the neutrino mass. CUORE will be made of $988 \mathrm{TeO}_{2}$ bolometers of $750 \mathrm{~g}$ each. Bolometers are detectors in which the energy from particle interactions is converted into heat and measured via the resulting rise in temperature. Operated at a temperature of about $10 \mathrm{mK}$, these detectors maintain an energy resolution of a few $\mathrm{keV}$ over their energy range, extending from a few $\mathrm{keV}$ up to several MeV. The measured resolution in the region of interest $(2527 \mathrm{keV})$ is about $5 \mathrm{keVFWHM}$; this, together with the low background and the high mass of the experiment, determines the sensitivity to the $0 v$ DBD. CUORE will be installed in the Gran Sasso National Laboratory (LNGS) in Italy and will start the data taking in about 2 years. The first CUORE tower, CUORE-0, composed by 52 bolometers, is under preparation and will start the data taking in the next year. A demonstrator experiment, CUORICINO, was operated in the same laboratory in the years 20032008. It was composed by $62 \mathrm{TeO}_{2}$ bolometers, for a total mass of $40.7 \mathrm{Kg}$. The acquired statistics was $19.75 \mathrm{~kg}\left({ }^{130} \mathrm{Te}\right) \cdot \mathrm{y}$, and the final result is presented in Fig. 1. No $0 v \mathrm{DBD}$ signal was found, the background level was $0.16 \pm 0.01$ counts $/ \mathrm{keV} / \mathrm{kg} / \mathrm{y}$ and the corresponding lower limit on the $0 v$ DBD half-life of ${ }^{130} \mathrm{Te}$ is $2.8 \times 10^{24} \mathrm{y}$ (90\% C.L.). This limit leads to an upper limit on the neutrino effective Majorana mass ranging from 0.3 to $0.7 \mathrm{eV}$, depending on the nuclear matrix elements considered in the computation.

CUORE could also search for dark matter (DM) interactions, provided that the energy threshold is sufficiently low. DM candidates such as weak interacting massive particles (WIMPs) and axion-like particles (ALPs) [2] are expected to produce signals at energies below $\sim 30 \mathrm{keV}$, and the interaction rate increases as the energy decreases. The energy threshold of CUORICINO bolometers, achieved using a trigger algorithm applied to the raw data samples, was of the order of tens of $\mathrm{keV}$. If the threshold were of few $\mathrm{keV}$, the CUORE experiment could be sensitive to DM interactions and thus play an important role in this growing research area. In the following we present

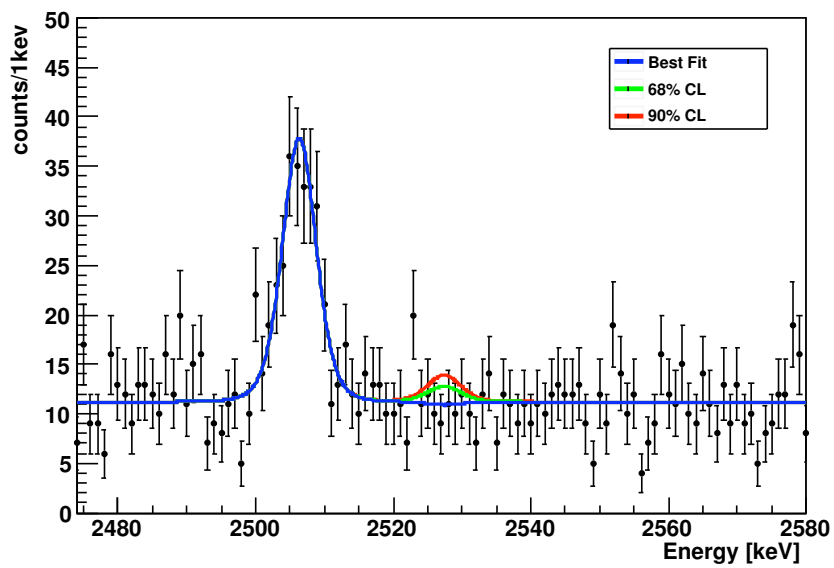

Figure 1: CUORICINO energy spectrum in the region of interest. The line at $2505 \mathrm{keV}$ is due to ${ }^{60} \mathrm{Co}$ contamination of the materials surrounding the bolometers. No peak is found at the expected $0 v \mathrm{DBD}$ energy of $2527 \mathrm{keV}$. 
a method we developed to lower the energy threshold, and the projected CUORE sensitivity to WIMP spin independent interactions.

\section{Experimental setup}

A CUORE bolometer is composed of two main parts, a $\mathrm{TeO}_{2}$ crystal and a neutron transmutation doped Germanium (NTD-Ge) thermistor [3]. The crystal is cube-shaped $\left(5 \times 5 \times 5 \mathrm{~cm}^{3}\right)$ and held by Teflon supports in copper frames. The frames are connected to the mixing chamber of a dilution refrigerator, which keeps the system at the temperature of $\sim 10 \mathrm{mK}$. The thermistor is glued to the crystal and acts as thermometer. When energy is released in the crystal, its temperature increases and changes the thermistor's resistance. The thermistor is biased with a constant current, and the voltage across it constitutes the signal [4]. Usually, a Joule heater is also glued to the crystal. It is used to inject controlled amounts of energy into the crystal, to emulate signals produced by particles [5]. The signal is amplified, filtered with a 6-pole active Bessel filter with a cut-off frequency ranging between 12 and $20 \mathrm{~Hz}$, and then acquired with an 18-bit ADC with a sampling frequency of $125 \mathrm{~Hz}$.

The data presented in this paper comes from a four-bolometers array operated by the CUORE collaboration at LNGS in 2009. The main purpose of the detector was to check one of the first production batches of CUORE crystals [6]. Thermistors were glued on all crystals, while heaters were glued only on two crystals. A typical signal produced by an $88 \mathrm{keV} \gamma$ particle generated by a ${ }^{127 m}$ Te de-excitation in a crystal is shown in Fig. 2.

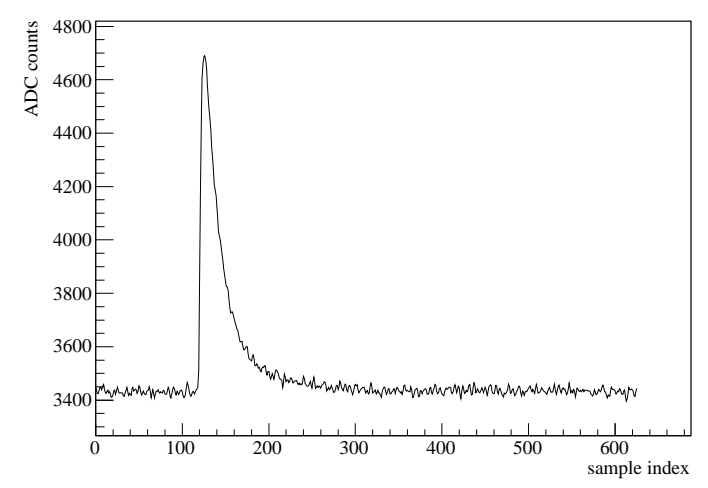

Figure 2: Signal produced by an $88 \mathrm{keV} \gamma$ particle fully absorbed in one detector. The signal is sampled at $125 \mathrm{~Hz}$ and the length of the window corresponds to $5.008 \mathrm{~s}$.

\section{Lowering the energy threshold}

At low energies the detection capability is limited by the detector noise. Electronics spikes, mechanical vibrations, and temperature fluctuations can produce pulses that, if not properly identified, generate nonphysical background. The lower the energy released in the bolometer, the more difficult it is to discriminate between physical and nonphysical pulses. 
We developed a trigger and a pulse shape identification algorithm [7] that operate on data samples processed using the matched filter technique [8]. This filter is designed to estimate the amplitude of a signal, maximizing the signal to noise ratio. Some slices of the filtered data are shown in Fig. 3. In it one can see that the noise fluctuations are reduced and signals with shape different from the expected are suppressed.
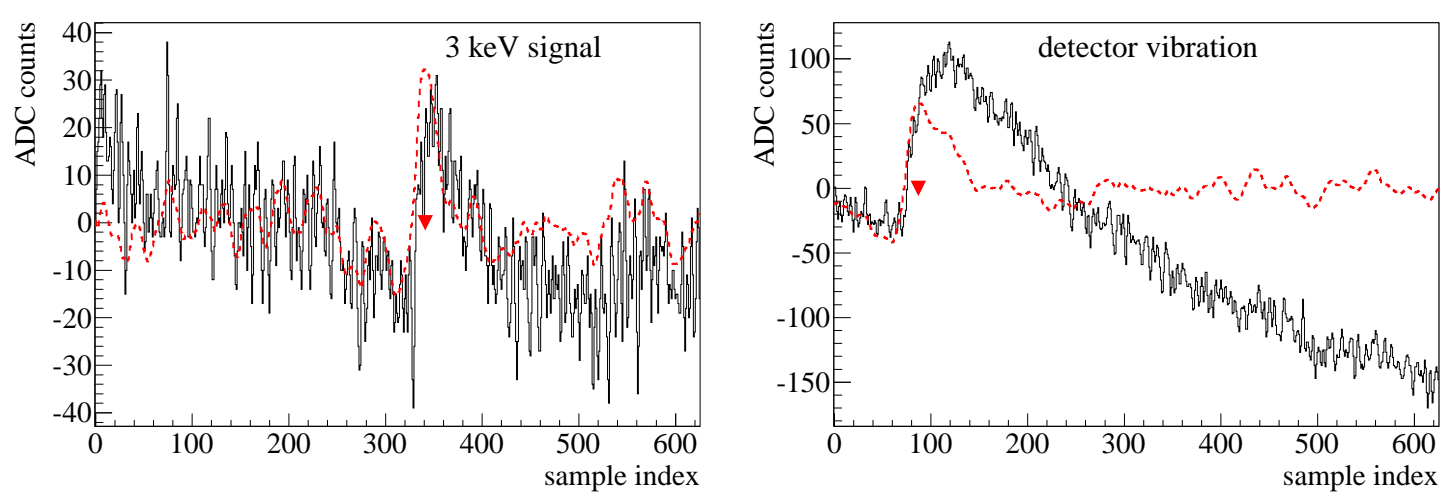

Figure 3: Windows of data samples containing pulses. The raw data (solid black line) have been shifted such that the first sample has zero ADC counts; the filtered data (dashed red line) are not modified. Red triangles identify the pulses detected by the trigger algorithm. The filter removes the baseline drifts and suppresses pulses with different shapes than the expected signal.

\subsection{Detection efficiency}

Pulses are triggered when the data samples exceed a positive threshold, and a new trigger is possible when the data samples return below threshold, or after that a local maximum is found. The detection efficiency is estimated on pulses generated by the heater, performing an energy scan from about $1 \mathrm{keV}$ up to $100 \mathrm{keV}$. In the case of bolometers without the heater, the estimation is made on simulated data. In Fig. 4 the efficiency as a function of energy is shown for a bolometer with the heater, and is compared with simulations of heater and particle pulses. In it, one can see that the simulation agrees well with data, validating the efficiency estimation on bolometers without the heater. The efficiency reaches a plateau of $\sim 91 \%$ at $E \sim 3 \mathrm{keV}$, which is the software energy threshold we set in the data analysis. Three bolometers has a $3 \mathrm{keV}$ energy threshold and a detection efficiency in excess of $80 \%$, while the fourth has a threshold of $\sim 10 \mathrm{keV}$. To ease the analysis job we ignored the fourth bolometer.

\subsection{Pulse shape identification}

To remove nonphysical pulses we fit the filtered pulses with the expected shape of the filtered signal. The $\chi^{2} /$ ndf of the fit is used as shape parameter, and the distribution obtained for one of the bolometers is shown in Fig. 5. In it, one can see that nonphysical pulses are very well separated. We apply a cut on this variable to select signal events in the energy region below $30 \mathrm{keV}$. The probability of loosing a signal event after this cut has been estimated to be less than $3 \%$. 


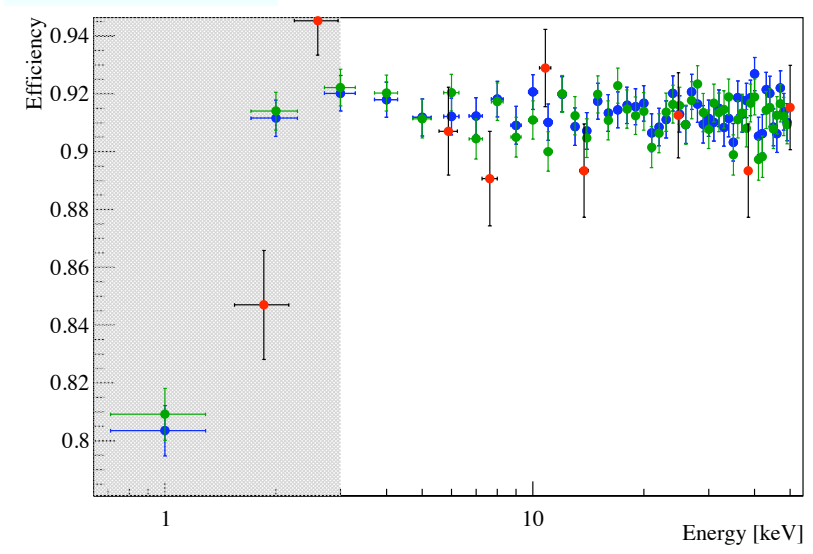

Figure 4: Detection efficiency as a function of energy for one of the four bolometers. The red dots represent the detection efficiencies on pulses generated by the heater, the blue (green) dots are simulated heater (particle) pulses. The plateau is reached at $\sim 3 \mathrm{keV}$, value which determines the energy threshold used in the data analysis. Above this threshold simulated data agrees well with real data, validating the efficiency estimation on bolometers without the heater, where only the simulation can be used.

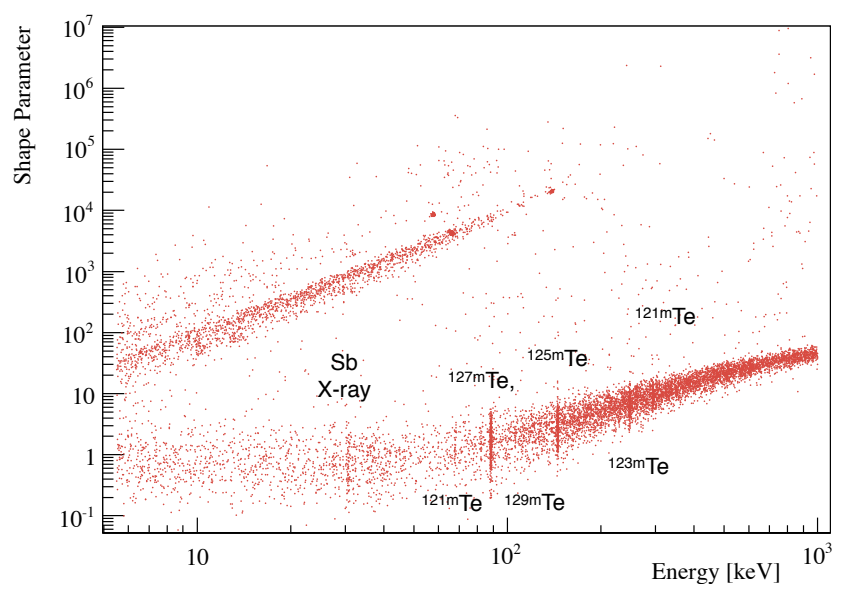

Figure 5: Shape parameter distribution of all the events in the range 5-1000 keV for one of the three bolometers used in the data analysis. The band at low values of the shape parameter is populated by signal events, where the lines generated by metastable Tellurium isotopes are visible. At higher values there are triggered mechanical vibrations and spikes.

\section{Sensitivity to dark matter interactions}

The four bolometers array took 19 days of data. The low energy spectrum obtained combining the three bolometers having $3 \mathrm{keV}$ threshold, after cutting on the shape parameter and correcting for the detection efficiencies, is shown in Fig. 6. The data in the range 3-5 keV are not displayed, since they are still being studied; in place of the data we show their approximate behavior, that is an exponential decay. The counting rate ranges from $\sim 20 \mathrm{cpd} / \mathrm{keV} / \mathrm{kg}$ at $3 \mathrm{keV}$ to $\sim 2 \mathrm{cpd} / \mathrm{keV} / \mathrm{kg}$ at $25 \mathrm{keV}$, and is well described by a double exponential decay. sWe estimate the CUORE sensitivity to WIMPs assuming that the background differential rate will be equal to the measured 


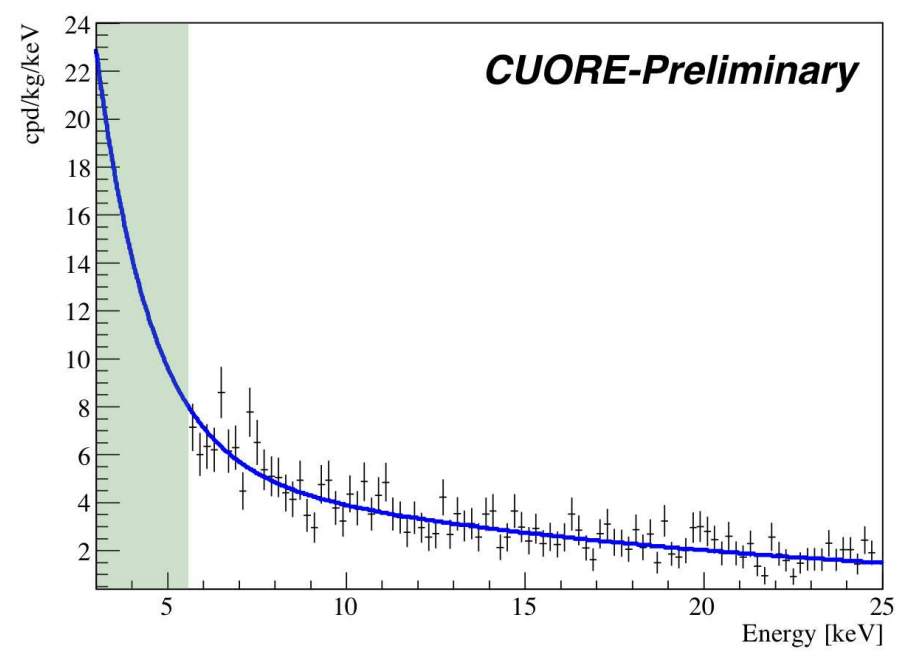

Figure 6: Differential rate at low energies obtained combining data from the three bolometers having the energy threshold at $3 \mathrm{keV}$. The region below $5 \mathrm{keV}$ is not shown, since it is still under study (see text). The data are fitted with a double exponential decay, and the fit function is used as model of the CUORE background.

value in the four bolometers array, and that all bolometers will have a $3 \mathrm{keV}$ threshold. It has to be stressed that CUORE bolometers, unlike many other detectors, cannot distinguish nuclear recoils from $\beta / \gamma$ interactions. Dark matter candidates like WIMPs, for example, are expected to induce nuclear recoils, so that experiments able to reject other types of interactions have a higher discovery potential. Nevertheless CUORE, thanks to its mass and to the low background expected, could detect dark matter searching for an annual modulation of the coutning rate at low energies.

We performed toy Monte Carlo simulations generating background events from the fit of the measured distribution in Fig. 6, and WIMP events from the theoretical distribution described in Ref. [9], using a quenching factor for nuclear recoils in $\mathrm{TeO}_{2}$ equal to 1 [10]. We included the dependence of the WIMP interaction rate on the time in the year, and estimated the background+signal asymmetry subtracting the 3-months integrated spectrum across 2 December from the 3-months integrated spectrum across 2 June. The $90 \%$ C.L. sensitivity to the cross-section for spin independent interactions normalized to nucleon, as a function of the WIMP mass, for CUORE-0 in 3 years and CUORE in 5 years of data taking, is shown in Fig. 7 together with the present results of the leading experiments of the field. As it can be seen from the figure, CUORE-0 and CUORE will not be as sensitive as experiments able to discriminate nuclear recoils. CUORE, however, could investigate the same parameter space of the DAMA/LIBRA experiment, and could be the only experiment other than DAMA/LIBRA looking for an annual modulation of dark matter interactions.

\section{References}

[1] R. Ardito et. al., CUORE: A cryogenic underground observatory for rare events, arXiv:hep-ex/0501010(2005) [hep-ex/0501010]. 


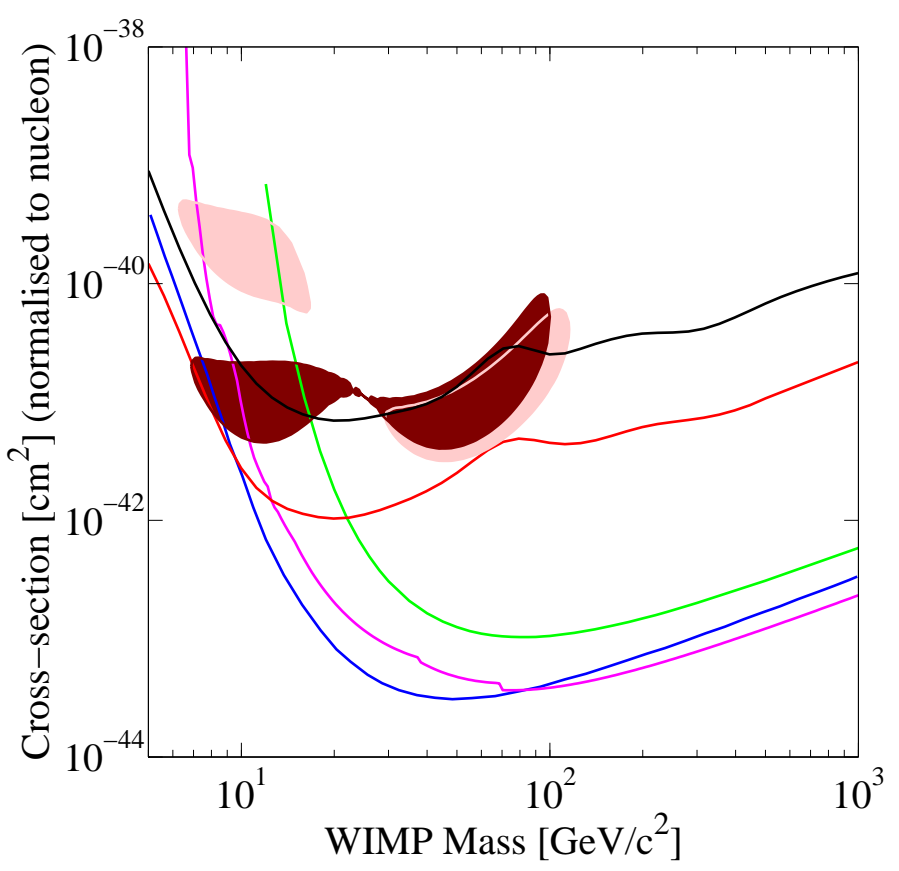

Figure 7: CUORE(5y) (in red) and CUORE-0(3y) (in black) expected sensitivities compared to the actual limits on WIMP-nucleon elastic scattering cross section. DAMA/LIBRA $3 \sigma$ evidence with ion channeling in dark red and without ion channeling in pink, Edelweiss II in green, XENON100 in blue, CDMS in magenta.

[2] G. Bertone, D. Hooper, and J. Silk, Particle dark matter: evidence, candidates and constraints, Phys. Rept. 405 (2005) 279, [hep-ph/ 0404175$].$

[3] K. M. Itoh et. al., Hopping conduction and metal-insulator transition in isotopically enriched neutron-transmutation-doped ${ }^{70}$ Ge:Ga, Phys. Rev. Lett. 77 (1996), no. 194058.

[4] C. Arnaboldi et. al., The programmable front-end system for CUORICINO, an array of large-mass bolometers, IEEE Trans. Nucl. Sci. 49 (2002) 2440.

[5] C. Arnaboldi, G. Pessina, and E. Previtali, A programmable calibrating pulse generator with multioutputs and very high stability, IEEE Trans. Nucl. Sci. 50 (2003) 979.

[6] C. Arnaboldi et. al., Production of high purity $\mathrm{TeO}_{2}$ single crystals for the study of neutrinoless double beta decay, J. Cryst. Growth 312 (2010), no. 202999.

[7] S. Di Domizio, F. Orio, and M. Vignati, Lowering the energy threshold of large-mass bolometric detectors, (arXiv expected by 10 Dec 2010).

[8] E. Gatti and P. F. Manfredi, Processing the signals from solid state detectors in elementary particle physics, Riv. Nuovo Cim. 9N1 (1986) 1.

[9] J. D. Lewin and P. F. Smith, Review of mathematics, numerical factors, and corrections for dark matter experiments based on elastic nuclear recoil, Astroparticle Physics 6 (1996), no. 187.

[10] A. Alessandrello et. al., The bolometers as nuclear recoil detectors, Nuclear Instruments and Methods in Physics Research Sec.A 409 (May, 1998) 451. 